Supporting Information

\title{
A Synthesis of L-(+)-Carbafuranomycin
}

\author{
Ja Young Lee, Volker Jäger* \\ [*] Institut für Organische Chemie, Universität Stuttgart, Pfaffenwaldring 55, \\ D-70569 Stuttgart, Germany \\ jager.ioc@po.uni-stuttgart.de
}


General Remarks: Solvents were purified and dried by standard methods. For column chromatography, silica gel (40 - $63 \mu \mathrm{m}$, Merck) was used, and for ion exchange purification acidic resin Dowex 50WX8 $\left(\mathrm{H}^{+}\right.$form, Fluka). Grignard reagents were purchased from Aldrich, lead tetraacetate from Fluka. Melting points were determined on a Fisher-Johns 4017 heating block and are uncorrected. ${ }^{1} \mathrm{H}$ and ${ }^{13} \mathrm{C}$ NMR spectra were recorded on Bruker AC 250, ARX 300, ARX 500 spectrometers using $\mathrm{Me}_{4} \mathrm{Si}$ as internal standard. IR spectra were recorded on a Perkin-Elmer 283 or a Bruker IFS 28 IR spectrometer. Mass spectra and high-resolution mass spectra (HRMS) were obtained with Finnigan quadrupole-MS 4500 and Finnigan MAT 95 spectrometers, respectively. Optical rotations were determined with a Perkin-Elmer polarimeter 241 MC. MPLC separations were carried out using a Lewa FL 1 pump, monitoring with a Knauer 97.00 diode array detector or a Knauer differential refractometer. The columns $(4 \mathrm{~cm} \times 40 \mathrm{~cm}, N=11600$, pressure $10-15$ bar, flow rate $40-60 \mathrm{~mL} / \mathrm{min}$ ) were packed with LiChroprep Si 60 silica gel (size $15-25 \mu \mathrm{m}$, Merck).

$(3 \mathrm{a} S, 6 \mathrm{a} S, 1$ 'S $) \quad$ and $\quad(3 \mathrm{a} R, 6 \mathrm{a} R, 1 ' S)-3-(1,2-O-$-Isopropylidenedioxyethyl)-4,6a-dihydro-3a $H$ cyclopenta[d]isoxazole (8) and (9):

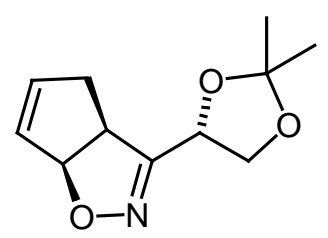

8

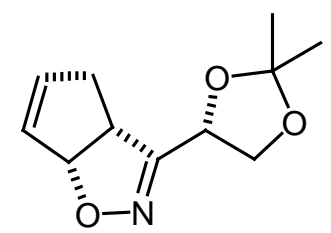

9

To a stirred solution of the hydroximoylchloride $\mathbf{6}(1.80 \mathrm{~g}, 10.0 \mathrm{mmol})$ in dry diethylether $(30 \mathrm{~mL})$ and freshly destilled cyclopentadiene $(10 \mathrm{~mL})$ under nitrogen $1_{\mathrm{M}}$ triethylamine solution $(12 \mathrm{~mL})$ in dry diethylether was added very slowly $(0.04 \mathrm{~mL} / \mathrm{min})$, using an motor-driven syringe. After completion the mixture was stirred for another $20 \mathrm{~h}$. Then water $(20 \mathrm{~mL})$ was added, the phase were separtaed, and the organic layer was washed with water $(20 \mathrm{~mL})$, dried $\left(\mathrm{MgSO}_{4}\right)$, and filtered. The solvent was evaporated in vacuo and the remaining orange-coloured oil was purified by flash chromatography on silica (petroleum ether/ethyl acetate 4:1) to afford the cyclopentaisoxazolines 8 and $9(1.68 \mathrm{~g}, 80 \%$, d. r. anti:syn = 56:44) as a colourless oil. The two isomer $\mathbf{8}$ and $\mathbf{9}$ showed the difficulties to separate, therefore the protecting group was changed in the next step. $\mathrm{C}_{11} \mathrm{H}_{15} \mathrm{NO}_{3}$ (209.2): calcd. C 63.14, H 7.23, N 6.69; found C 62.83, H 7.23, N 6.73. 
(3aS,6aS,1'S)- $\quad$ and $\quad(3 a R, 6 a R, 1 ' S)-3-(1,2-B i s-b e n z y l o x y-e t h y l)-4,6 a-d i h y d r o-3 a H-$ cyclopenta[d]isoxazole (10) and (11):

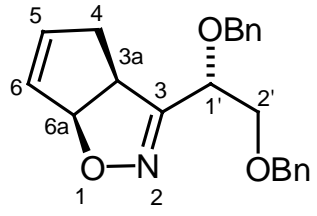

10

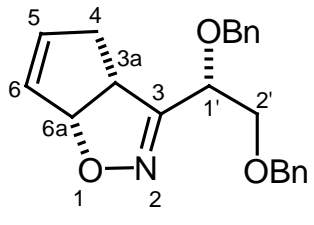

11

To a stirred solution of the cyclopentaisoxazolines 8 and $9(1.00 \mathrm{~g}, 4.78 \mathrm{mmol})$ in methanol $(4 \mathrm{~mL})$ and water $(1 \mathrm{~mL})$ was added trifluoracetic acid $(0.15 \mathrm{~mL})$ at room temperature. After standing for $1 \mathrm{~d}$, TLC analysis indicated that the reaction had gone to completion. After neutralisation with $1 \mathrm{~N}$ solution of ammonia $(4 \mathrm{~mL})$ silica gel $(0.5 \mathrm{~g})$ was added to the mixture and the solvent was evaporated. The product absorbed on silica was loaded onto the top of a column of silica gel and eluted with ethyl acetate. Removal of the solvent in vacuo afforded the diols (761 mg, $94 \%$ ) as a colourless oil. Benzylation and diastereomer separation: Sodium hydride (325 mg, $13.5 \mathrm{mmol}$ ) and benzyl bromide $(1.61 \mathrm{~mL}, 13.5 \mathrm{mmol})$ were dissolved in dry DMF $(8 \mathrm{~mL})$, to which the solution of diols $(761 \mathrm{mg}, 4.50 \mathrm{mmol})$ in dry DMF $(7 \mathrm{~mL})$ was added dropwise. After stirring for $4 \mathrm{~h}$, the reaction was quenched at $0{ }^{\circ} \mathrm{C}$ by addition of methanol $(5 \mathrm{~mL})$, saturated aqueous $\mathrm{NH}_{4} \mathrm{Cl}(20 \mathrm{~mL})$, and water $(10 \mathrm{~mL})$. The mixture was extracted with $\mathrm{CH}_{2} \mathrm{Cl}_{2}(4 \times 20 \mathrm{~mL})$. The combined organic solutions were washed with a saturated aqueous solution of $\mathrm{LiCl}(10 \mathrm{~mL})$ and water $(2 \times 10 \mathrm{~mL})$ and then dried $\left(\mathrm{MgSO}_{4}\right)$. After concentration of the solvent in vacuo, the residue was filtered over a pad of silica gel (petroleum ether/ethyl acetate 4:1) and again the solvent was removed under reduced pressure. Then, the diastereomers $\mathbf{1 0}$ and $\mathbf{1 1}$ were separated by MPLC (petroleum ether/ethyl acetate 4:1) affording $\mathbf{1 0}$ (anti, $687 \mathrm{mg}, 41 \%$ ) and $\mathbf{1 1}(\mathrm{syn}, 611 \mathrm{mg}, 37 \%$ ) as colourless oils. 10 (anti): $[\alpha]_{D}^{20}=-36.9\left(c=1.20, \mathrm{CHCl}_{3}\right)$. IR (film): $\tilde{v}=2944,2920,2861,1496,1452,1126$, 1102, 1086, 742, $695 \mathrm{~cm}^{-1} .{ }^{1} \mathrm{H}$ NMR $\left(500.2 \mathrm{MHz}, \mathrm{CDCl}_{3}\right): \delta=2.53\left(\mathrm{dd}, J_{3 \mathrm{a}, 4 \mathrm{a}}=8.3, J_{4 \mathrm{a}, 4 \mathrm{~b}}=17.6\right.$ $\left.\mathrm{Hz}, 1 \mathrm{H}, 4-\mathrm{H}_{\mathrm{a}}\right), 2.84\left(\mathrm{dd}, J_{3 \mathrm{a}, 4 \mathrm{~b}}=1.9, J_{4 \mathrm{a}, 4 \mathrm{~b}}=17.6 \mathrm{~Hz}, 1 \mathrm{H}, 4-\mathrm{H}_{\mathrm{b}}\right), 3.79\left(\mathrm{~A}\right.$ of dAB, $J_{1^{\prime}, 2^{\prime} \mathrm{a}}=5.9, J_{\mathrm{A}, \mathrm{B}}$ $\left.=10.3 \mathrm{~Hz}, 1 \mathrm{H}, 2^{\prime}-\mathrm{H}_{\mathrm{a}}\right), 3.82\left(\mathrm{~B}\right.$ of dAB, $\left.J_{1^{\prime}, 2^{\prime} \mathrm{b}}=6.1, J_{\mathrm{A}, \mathrm{B}}=10.3 \mathrm{~Hz}, 1 \mathrm{H}, 2^{\prime}-\mathrm{H}_{\mathrm{b}}\right), 3.87\left(\mathrm{~d}^{\prime \prime} \mathrm{t}^{\prime}\right.$, $J_{3 \mathrm{a}, 4 \mathrm{~b}}=$ $\left.1.8, J_{3 \mathrm{a}, 4 \mathrm{a}}=J_{3 \mathrm{a}, 6 \mathrm{a}}=8.7 \mathrm{~Hz}, 1 \mathrm{H}, 3 \mathrm{a}-\mathrm{H}\right), 4.48\left(\mathrm{~A}^{\prime}\right.$ of $\left.\mathrm{A}^{\prime} \mathrm{B}^{\prime}, J_{\mathrm{A}^{\prime}, \mathrm{B}^{\prime}}=11.7 \mathrm{~Hz}, 1 \mathrm{H}, \mathrm{OC}_{\mathrm{A}_{\mathrm{A}}} \cdot \mathrm{H}_{\mathrm{B}}, \mathrm{C}_{6} \mathrm{H}_{5}\right)$,

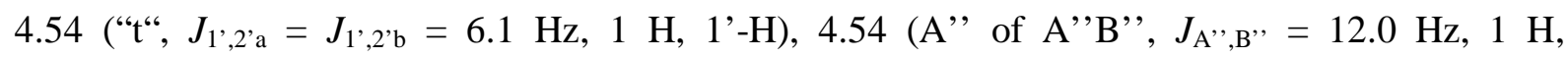

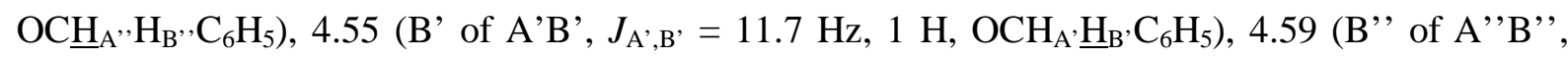
$\left.J_{\mathrm{A}^{\prime}, \mathrm{B}^{\prime}}=12.0 \mathrm{~Hz}, 1 \mathrm{H}, \mathrm{OCH}_{\mathrm{A}}{ }^{\prime} \underline{\mathrm{H}}_{\mathrm{B}}{ }, \mathrm{C}_{6} \mathrm{H}_{5}\right), 5.67\left(\mathrm{dd}, J_{3 \mathrm{a}, 6 \mathrm{a}}=9.2, J_{5,6 \mathrm{a}}=1.2 \mathrm{~Hz}, 1 \mathrm{H}, 6 \mathrm{a}-\mathrm{H}\right), 5.81(\mathrm{~d}$, $\left.J_{5,6}=6.0 \mathrm{~Hz}, 1 \mathrm{H}, 6-\mathrm{H}\right), 5.97\left(\mathrm{dd}, J_{5,6}=5.8, J_{5,6 \mathrm{a}}=1.2 \mathrm{~Hz}, 1 \mathrm{H}, 5-\mathrm{H}\right), 7.23-7.35\left(\mathrm{~m}, 10 \mathrm{H}, 2 \mathrm{C}_{6} \mathrm{H}_{5}\right)$ ppm. ${ }^{13} \mathrm{C}$ NMR (125.8 MHz, $\left.\mathrm{CDCl}_{3}\right): \delta=36.0$ (C-4), 49.7 (C-3a), 70.7 (C-2'), 71.6 (C-1'), 73.5, 
$73.9\left(2 \mathrm{OCH}_{2} \mathrm{C}_{6} \mathrm{H}_{5}\right), 91.3$ (C-6a), 128.2, 128.3, $128.5,128.9\left(o-, m-, p-\mathrm{C}\right.$ of $\left.\mathrm{C}_{6} \mathrm{H}_{5}\right), 129.9,134.5$ (C5, C-6), 138.0, $138.2\left(i-\mathrm{C}\right.$ of $\left.\mathrm{C}_{6} \mathrm{H}_{5}\right), 157.5$ (C-3) ppm. $\mathrm{C}_{22} \mathrm{H}_{23} \mathrm{NO}_{3}$ (349.4): calcd. C 75.62, H 6.64, N 4.01; found C 75.52, H 6.65, N 3.96.

11 (syn): $[\alpha]_{D}^{20}=+135.3\left(c=1.14, \mathrm{CHCl}_{3}\right)$. IR (film): $\tilde{v}=2935,2926,2860,2364,2328,1452$, 1126, 1101, 882, 743, 695. ${ }^{1} \mathrm{H}$ NMR (500.2 MHz, $\left.\mathrm{CDCl}_{3}\right): \delta=2.58\left(\mathrm{dd}^{\mathrm{a}} \mathrm{t}\right.$ ”, $J_{3 \mathrm{a}, 4 \mathrm{a}}=7.9, J_{4 \mathrm{a}, 4 \mathrm{~b}}=17.7$, $\left.J_{4 \mathrm{a}, 5}=J_{4 \mathrm{a}, 6}=2.3 \mathrm{~Hz}, 1 \mathrm{H}, 4-\mathrm{H}_{\mathrm{a}}\right), 2.64\left(\mathrm{dd}, J_{3 \mathrm{a}, 4 \mathrm{~b}}=2.9, J_{4 \mathrm{a}, 4 \mathrm{~b}}=17.7,1 \mathrm{H}, 4-\mathrm{H}_{\mathrm{b}}\right), 3.74(\mathrm{~A}$ of dAB , $\left.J_{1^{\prime}, 2^{\prime} \mathrm{a}}=5.2, J_{\mathrm{A}, \mathrm{B}}=10.3 \mathrm{~Hz}, 1 \mathrm{H}, 2^{\prime}-\mathrm{H}_{\mathrm{a}}\right), 3.79\left(\mathrm{~B}\right.$ of dAB, $\left.J_{1^{\prime}, 2^{\prime} \mathrm{b}}=6.7, J_{\mathrm{A}, \mathrm{B}}=10.3 \mathrm{~Hz}, 1 \mathrm{H}, 2^{\prime}-\mathrm{H}_{\mathrm{b}}\right)$, $3.85\left(\mathrm{dddd}, J_{3 \mathrm{a}, 4 \mathrm{a}}=7.9, J_{3 \mathrm{a}, 4 \mathrm{~b}}=3.1, J_{3 \mathrm{a}, 6 \mathrm{a}}=9.1, J_{3 \mathrm{a}, 1}{ }^{\prime}=0.68 \mathrm{~Hz}, 1 \mathrm{H}, 3 \mathrm{a}-\mathrm{H}\right), 4.53\left(\mathrm{~A}^{\prime}\right.$ of $\mathrm{A}^{\prime} \mathrm{B}^{\prime}, J_{\mathrm{A}^{\prime}, \mathrm{B}^{\prime}}$ $\left.=11.9 \mathrm{~Hz}, 1 \mathrm{H}, \mathrm{OC}_{\mathrm{H}}{ }^{\prime} \mathrm{H}_{\mathrm{B}}, \mathrm{C}_{6} \mathrm{H}_{5}\right), 4.57\left(\mathrm{dd}, J_{1^{\prime}, 2^{\prime} \mathrm{a}}=5.1, J_{1^{\prime}, 2^{\prime} \mathrm{b}}=6.7 \mathrm{~Hz}, 1 \mathrm{H}, 1^{\prime}-\mathrm{H}\right), 4.57\left(\mathrm{~A}^{\prime}\right.$ ' of A' $\left.{ }^{\prime}{ }^{\prime},, J_{\mathrm{A}^{\prime}, \mathrm{B}}{ }^{\prime}=11.9 \mathrm{~Hz}, 1 \mathrm{H}, \mathrm{OC}_{\mathrm{A}}, \mathrm{H}_{\mathrm{B}}, \mathrm{C}_{6} \mathrm{H}_{5}\right), 4.57\left(\mathrm{~B}^{\prime}\right.$ of $\mathrm{A}^{\prime} \mathrm{B}^{\prime}, J_{\mathrm{A}^{\prime}, \mathrm{B}},=12.1 \mathrm{~Hz}, 1 \mathrm{H}$, $\left.\mathrm{OCH}_{\mathrm{A}} \underline{\mathrm{H}}_{\mathrm{B}}, \mathrm{C}_{6} \mathrm{H}_{5}\right), 4.57$ (B', of A' 'B', $\left.J_{\mathrm{A}^{\prime}, \mathrm{B}^{\prime}}=11.9 \mathrm{~Hz}, 1 \mathrm{H}, \mathrm{OCH}_{\mathrm{A}}, \underline{\mathrm{H}}_{\mathrm{B}}, \mathrm{C}_{6} \mathrm{H}_{5}\right), 5.63\left(\mathrm{dd}, J_{3 \mathrm{a}, 6 \mathrm{a}}=\right.$ 9.4, $\left.J_{5,6 \mathrm{a}}=1.1 \mathrm{~Hz}, 1 \mathrm{H}, 6 \mathrm{a}-\mathrm{H}\right), 5.82\left(\mathrm{dd}, J_{4 \mathrm{a}, 6}=2.1, J_{5,6}=5.7 \mathrm{~Hz}, 1 \mathrm{H}, 6-\mathrm{H}\right), 5.93\left(\mathrm{ddd}, J_{4 \mathrm{a}, 5}=2.3\right.$, $\left.J_{5,6}=5.7, J_{5,6 \mathrm{a}}=1.1 \mathrm{~Hz}, 1 \mathrm{H}, 5-\mathrm{H}\right), 7.24-7.35\left(\mathrm{~m}, 10 \mathrm{H}, 2 \mathrm{C}_{6} \mathrm{H}_{5}\right) \mathrm{ppm} .{ }^{13} \mathrm{C} \mathrm{NMR}(125.8 \mathrm{MHz}$, $\left.\mathrm{CDCl}_{3}\right): \delta=36.3(\mathrm{C}-4), 49.7(\mathrm{C}-3 \mathrm{a}), 71.4\left(\mathrm{C}-2\right.$ ') $71.7(\mathrm{C}-1$ ') $)$ 73.3, $74.3\left(2 \mathrm{OCH}_{2} \mathrm{C}_{6} \mathrm{H}_{5}\right), 90.9(\mathrm{C}-$ 6a), 127.7, 127.8, 127.9, 128.4, $128.4\left(o-, m-, p-\mathrm{C}\right.$ of $\left.\mathrm{C}_{6} \mathrm{H}_{5}\right), 129.2,134.1$ (C-5, C-6), 137.6, 137.8 (i-C of $\mathrm{C}_{6} \mathrm{H}_{5}$ ), 158.4 (C-3) ppm. $\mathrm{C}_{22} \mathrm{H}_{23} \mathrm{NO}_{3}$ (349.4): calcd. C 75.62, H 6.64, N 4.01; found C 75.82, H 6.65, N 3.99. 

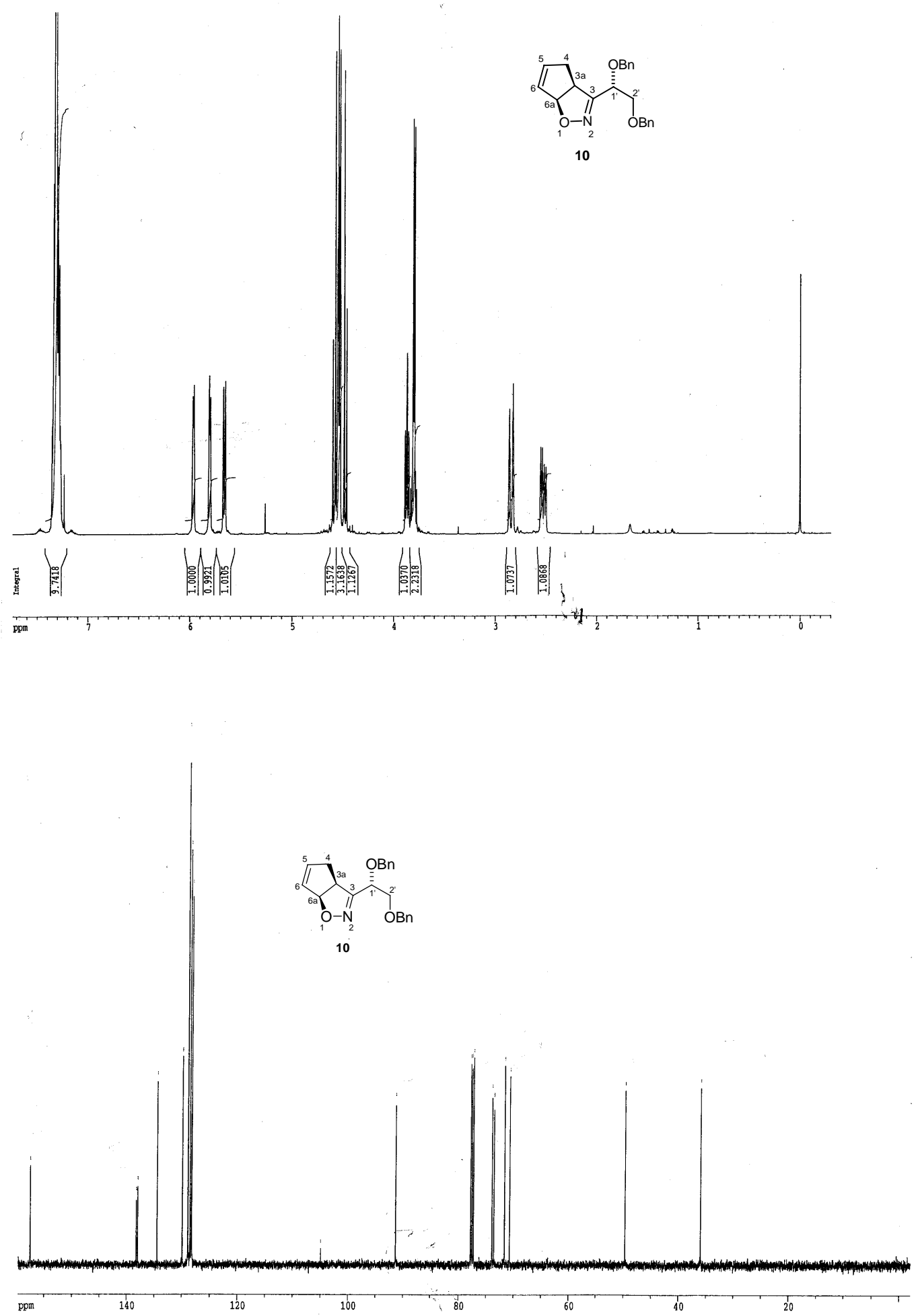


\section{(1S,5S,1'S,2'S)-5-[1-tert-Butoxycarbonylamino-2,3-bis-(benzyloxy)-propyl]-cyclopent-2-enol}

(12):

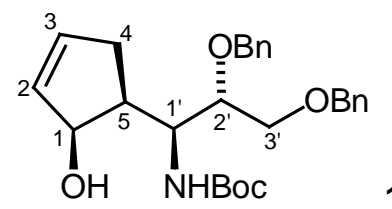

12

$\mathrm{LiAlH}_{4}(95 \mathrm{mg}, 2.5 \mathrm{mmol})$ under nitrogen was suspended in dry diethylether $(20 \mathrm{~mL})$ in a flamedried flask. The mixture was cooled to $0{ }^{\circ} \mathrm{C}$ and the solution of 10 (349 $\left.\mathrm{mg}, 1.00 \mathrm{mmol}\right)$ in dry diethylether $(5 \mathrm{~mL})$ was added dropwise. The cooling bath was removed and the reaction mixture was heated under reflux for $1.5 \mathrm{~h}$. The reaction mixture was cooled down and then hydrolysed with water $(0.05 \mathrm{~mL})$, a $20 \%$ aqueous solution of $\mathrm{NaOH}(0.1 \mathrm{~mL})$, and water $(0.3 \mathrm{~mL})$ at $0{ }^{\circ} \mathrm{C}$ and stirred additionally for $30 \mathrm{~min}$. After addition of $\mathrm{MgSO}_{4}(5 \mathrm{~g})$, stirring was continued for $16 \mathrm{~h}$ at room temp.. The mixture was filtered and the filter cake was washed thoroughly with $\mathrm{CH}_{2} \mathrm{Cl}_{2}$ (200 $\mathrm{mL}$ ). After evaporation of the solvent, a colourless oil was obtained (d.r. 90:10), which was dissolved in dioxane/water $(3: 1,4 \mathrm{~mL})$ and treated with di-tert-butyldicarbonate $(546 \mathrm{mg}, 2.50$ mmol) at $0{ }^{\circ} \mathrm{C}$. The reaction mixture was stirred at room temp. for $16 \mathrm{~h}$, then water $(30 \mathrm{~mL})$ was added and the mixture was extracted with $\mathrm{CH}_{2} \mathrm{Cl}_{2}(4 \times 20 \mathrm{~mL})$ and the organic solutions were dried $\left(\mathrm{MgSO}_{4}\right)$. After concentration of the solvent, the major diastereoisomer 12 was separated from the minor one by repeated (three times) flash chromatography on silica (petroleum ether/ethyl acetate $=$ 6:4) to afford amino alcohol 12 as a colourless oil $(352 \mathrm{mg}, 78 \%) .12:[\alpha]_{D}^{20}=+11.1(c=1.27$, $\mathrm{CHCl}_{3}$ ). IR (film): $\tilde{v}=3417,2975,2928,2866,1694,1504,1454,1095,1058,1027 \mathrm{~cm}^{-1} .{ }^{1} \mathrm{H} \mathrm{NMR}$ $\left(300.1 \mathrm{MHz}, \mathrm{CD}_{3} \mathrm{OD}\right): \delta=1.42\left[\mathrm{~s}, 9 \mathrm{H}, \mathrm{C}\left(\mathrm{CH}_{3}\right)_{3}\right], 2.16-2.32\left(\mathrm{~m}, 3 \mathrm{H}, 5-\mathrm{H}, 4-\mathrm{H}_{\mathrm{a}}, 4-\mathrm{H}_{\mathrm{b}}\right), 3.70(\mathrm{dd}$, $\left.J_{2^{\prime}, 3^{\prime} \mathrm{a}}=5.3, J_{3^{\prime} \mathrm{a}, 3^{\prime} \mathrm{b}}=10.9 \mathrm{~Hz}, 1 \mathrm{H}, 3^{\prime}-\mathrm{H}_{\mathrm{a}}\right), 3.79\left(\mathrm{dd}, J_{2^{\prime}, 3^{\prime} \mathrm{b}}=3.0, J_{3^{\prime} \mathrm{a}, 3^{\prime} \mathrm{b}}=10.9 \mathrm{~Hz}, 1 \mathrm{H}, 3^{\prime}-\mathrm{H}_{\mathrm{b}}\right), 3.92$ $\left(\mathrm{dd}, J_{2^{\prime}, 3^{\prime} \mathrm{a}}=5.3, J_{2^{\prime}, 3^{\prime} \mathrm{b}}=3.0 \mathrm{~Hz}, 1 \mathrm{H}, 2^{\prime}-\mathrm{H}\right), 4.27\left(\mathrm{~m}, 1 \mathrm{H}, 1^{\prime}-\mathrm{H}\right), 4.45$ (m, $\left.1 \mathrm{H}, 1-\mathrm{H}\right), 4.53$ (s, $2 \mathrm{H}$, $\left.\mathrm{OC}_{2} \mathrm{C}_{6} \mathrm{H}_{5}\right), 4.58\left(\mathrm{~A}\right.$ of $\left.\mathrm{AB}, J_{\mathrm{A}, \mathrm{B}}=11.4 \mathrm{~Hz}, 1 \mathrm{H}, \underline{\mathrm{C}}_{\mathrm{A}} \mathrm{CH}_{\mathrm{B}} \mathrm{C}_{6} \mathrm{H}_{5}\right), 4.72\left(\mathrm{~B}\right.$ of $\mathrm{AB}, J_{\mathrm{A}, \mathrm{B}}=11.4 \mathrm{~Hz}, 1$ $\mathrm{H}, \mathrm{CH}_{\mathrm{A}} \underline{\mathrm{CH}}_{\mathrm{B}} \mathrm{C}_{6} \mathrm{H}_{5}$ ), 5.84 (d, $J_{2,3}=5.7 \mathrm{~Hz}, 1 \mathrm{H}, 2-\mathrm{H}$ od. 3-H), 6.00 (d, $J_{2,3}=5.7 \mathrm{~Hz}, 1 \mathrm{H}, 2-\mathrm{H}$ od. 3$\mathrm{H}), 6.44\left(\mathrm{bd}, J_{1}{ }^{\prime}, \mathrm{NH}=10.1 \mathrm{~Hz}, 1 \mathrm{H}, \mathrm{NH}\right), 7.20-7.43\left(\mathrm{~m}, 10 \mathrm{H}, 2 \mathrm{C}_{6} \mathrm{H}_{5}\right) \mathrm{ppm} .{ }^{13} \mathrm{C} \mathrm{NMR}(125.8 \mathrm{MHz}$, $\left.\mathrm{CDCl}_{3}\right): \delta=28.4\left[\mathrm{C}\left(\underline{\mathrm{CH}}_{3}\right)_{3}\right], 34.1$ (C-4), 44.0 (C-5), 50.3 (C-1'), 71.2, 71.8, 73.8 (C-3', 2 $\left.\underline{\mathrm{C}}_{2} \mathrm{C}_{6} \mathrm{H}_{5}\right), 76.4$ (C-2'), 78.9 (C-1), $79.3\left[\underline{\mathrm{C}}\left(\mathrm{CH}_{3}\right)_{3}\right], 127.7,127.8,128.0,128.4,128.5$ (o-, m-, $p$-C of $\mathrm{C}_{6} \mathrm{H}_{5}$ ), 133.6, 133.9 (C-2 and C-3), 137.8, 138.3 ( $i$-C of $\mathrm{C}_{6} \mathrm{H}_{5}$ ), 156.6 (1'-NHCO) ppm. $\mathrm{C}_{27} \mathrm{H}_{35} \mathrm{NO}_{5}$ (453.6): calcd. C 71.49, H 7.78, N 3.09; found C 71.58, H 7.89, N 3.04. 


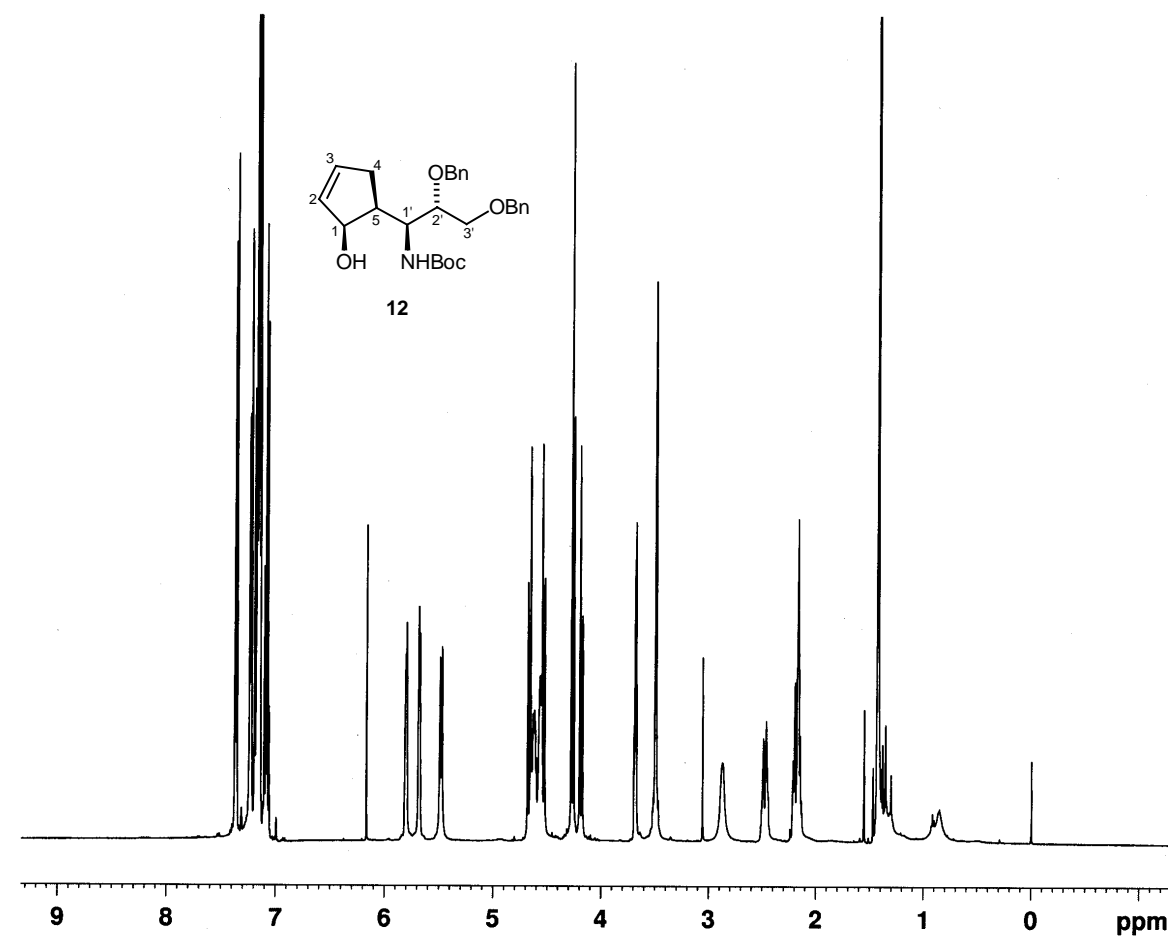




\section{$(1 S, 5 S, 1 ' S, 2$ 'S $)$-1-Acetoxy-5-[2,3-bis-(benzyloxy)-1-tert-butoxycarbonylaminopropyl]-2-cyclo-}

pentene (13):

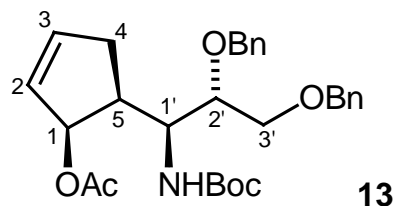

To a stirred solution of the $N$-Boc-amino alcohol $12(122 \mathrm{mg}, 0.269 \mathrm{mmol})$ in dry pyridine $(5 \mathrm{~mL})$ was added acetic anhydride $(51 \mu \mathrm{l}, 0.54 \mathrm{mmol})$ and then DMAP (ca. $2 \mathrm{mg}$ ) under nitrogen. After stirring for $16 \mathrm{~h}$, TLC analysis indicated that the reaction has gone to completion. $1 \mathrm{~N} \mathrm{HCl}$ solution $(15 \mathrm{~mL})$ was added at $0{ }^{\circ} \mathrm{C}$ and the mixture was then extracted with $\mathrm{CH}_{2} \mathrm{Cl}_{2}(4 \times 20 \mathrm{~mL})$. The combined solutions were washed with saturated aqueous $\mathrm{NaHCO}_{3}$ and dried $\left(\mathrm{MgSO}_{4}\right)$. After concentration of the solvent, the residue was purified by flash chromatography (petroleum ether/ethyl acetate 4:1) to afford the acetate $X(130 \mathrm{mg}, 97 \%)$ as a colourless oil. 13: $[\alpha]_{D}^{20}=+102.0$ $\left(c=1.00, \mathrm{CHCl}_{3}\right) . \mathrm{IR}\left(\right.$ film): $\tilde{v}=1713,1488,1362,1229,1156,725,699,599 \mathrm{~cm}^{-1} .{ }^{1} \mathrm{H}$ NMR $\left(500.2 \mathrm{MHz}, \mathrm{CDCl}_{3}\right): \delta=1.45\left[\mathrm{~s}, 9 \mathrm{H}, \mathrm{C}\left(\mathrm{CH}_{3}\right)_{3}\right], 1.84\left(\mathrm{~s}, 3 \mathrm{H}, \mathrm{OCOCH}_{3}\right), 2.33(\mathrm{~m}, 1 \mathrm{H}, 5-\mathrm{H}), 2.40$ $\left(\mathrm{d}, J_{4 \mathrm{a}, 4 \mathrm{~b}}=17.0 \mathrm{~Hz}, 1 \mathrm{H}, 4-\mathrm{H}_{\mathrm{a}}\right), 2.51\left(\mathrm{~d}, J_{4 \mathrm{a}, 4 \mathrm{~b}}=17.0 \mathrm{~Hz}, 1 \mathrm{H}, 4-\mathrm{H}_{\mathrm{b}}\right), 3.53\left(\mathrm{~m}, 1 \mathrm{H}, 2^{\prime}-\mathrm{H}\right), 3.72(\mathrm{dd}$, $\left.J_{2^{\prime}, 3^{\prime} \mathrm{a}}=2.9, J_{3^{\prime} \mathrm{a}, 3^{\prime} \mathrm{b}}=11.0 \mathrm{~Hz}, 1 \mathrm{H}, 3^{\prime}-\mathrm{H}_{\mathrm{a}}\right), 3.75\left(\mathrm{dd}, J_{2^{\prime}, 3^{\prime} \mathrm{b}}=3.9, J_{3^{\prime} \mathrm{a}, 3^{\prime} \mathrm{b}}=11.0 \mathrm{~Hz}, 1 \mathrm{H}, 3^{\prime}-\mathrm{H}_{\mathrm{b}}\right), 4.41$ $\left(\mathrm{d}, J_{1}, \mathrm{NH}=10.0 \mathrm{~Hz}, 1 \mathrm{H}, 1^{\prime}-\mathrm{H}\right), 4.48\left(\mathrm{~A}\right.$ of $\left.\mathrm{AB}, J_{\mathrm{A}, \mathrm{B}}=12.0 \mathrm{~Hz}, 1 \mathrm{H}, \mathrm{CH}_{\mathrm{A}} \mathrm{CH}_{\mathrm{B}} \mathrm{C}_{6} \mathrm{H}_{5}\right), 4.54(\mathrm{~B}$ of $\mathrm{AB}$, $\left.J_{\mathrm{A}, \mathrm{B}}=12.0 \mathrm{~Hz}, 1 \mathrm{H}, \mathrm{CH}_{\mathrm{A}} \underline{\mathrm{CH}}_{\mathrm{B}} \mathrm{C}_{6} \mathrm{H}_{5}\right), 4.64\left(\mathrm{~A}^{\prime}\right.$ of $\left.\mathrm{A}^{\prime} \mathrm{B}^{\prime}, J_{\mathrm{A}^{\prime}, \mathrm{B}^{\prime}}=12.1 \mathrm{~Hz}, 1 \mathrm{H}, \mathrm{C}_{\mathrm{A}^{\prime}}, \mathrm{CH}_{\mathrm{B}}, \mathrm{C}_{6} \mathrm{H}_{5}\right), 4.72$ (B' of $\left.\mathrm{A}^{\prime} \mathrm{B}^{\prime}, J_{\mathrm{A}^{\prime}, \mathrm{B}}{ }^{\prime}=12.1 \mathrm{~Hz}, 1 \mathrm{H}, \mathrm{CH}_{\mathrm{A}}{ }^{\prime} \underline{\mathrm{C}}_{\mathrm{B}}, \mathrm{C}_{6} \mathrm{H}_{5}\right), 5.31(\mathrm{~m}, 1 \mathrm{H}, 1-\mathrm{H}), 5.39\left(\mathrm{~d}, J_{1^{\prime}, \mathrm{NH}}=10.2 \mathrm{~Hz}, 1\right.$ $\mathrm{H}, \mathrm{NH}), 5.89\left(\mathrm{~m}, 1 \mathrm{H}, 2 \mathrm{H}\right.$ or 3-H), 6.10 (d, $J_{2,3}=5.7 \mathrm{~Hz}, 1 \mathrm{H}, 2-\mathrm{H}$ or 3-H), $7.24-7.35$ (m, $10 \mathrm{H}, 2$ $\left.\mathrm{C}_{6} \mathrm{H}_{5}\right)$ ppm. ${ }^{13} \mathrm{C}$ NMR $\left(62.9 \mathrm{MHz}, \mathrm{CDCl}_{3}\right): \delta=21.1\left(\underline{\mathrm{CH}}_{3} \mathrm{COO}\right), 28.5\left[\mathrm{C}\left(\underline{\mathrm{CH}}_{3}\right)_{3}\right], 35.4(\mathrm{C}-4), 43.3$ (C-5), 50.6 (C-1'), 71.1, 71.2 (C-3' and $\left.\mathrm{CH}_{2} \mathrm{C}_{6} \mathrm{H}_{5}\right), 73.9\left(\mathrm{CH}_{2} \mathrm{C}_{6} \mathrm{H}_{5}\right), 77.0$ (C-2'), 78.1 (C-1), 79.0 $\left[\underline{\mathrm{C}}\left(\mathrm{CH}_{3}\right)_{3}\right], 127.7,127.8,127.9,127.9,128.3,128.4,128.5\left(o-, m-, p-\mathrm{C}\right.$ of $\left.\mathrm{C}_{6} \mathrm{H}_{5}\right), 129.7$ (C-3), 137.7 ( $i$-C of $\left.\mathrm{C}_{6} \mathrm{H}_{5}\right), 138.0(\mathrm{C}-2), 138.5\left(i-\mathrm{C}\right.$ of $\left.\mathrm{C}_{6} \mathrm{H}_{5}\right), 156.3$ (1'-NHCOO), 170.9 (2-OCOCH 3$)$ ppm. $\mathrm{C}_{29} \mathrm{H}_{37} \mathrm{NO}_{6}$ (495.6): calcd. C 70.28, H 7.53, N 2.83; found C 70.00, H 7.61, N 2.68. 

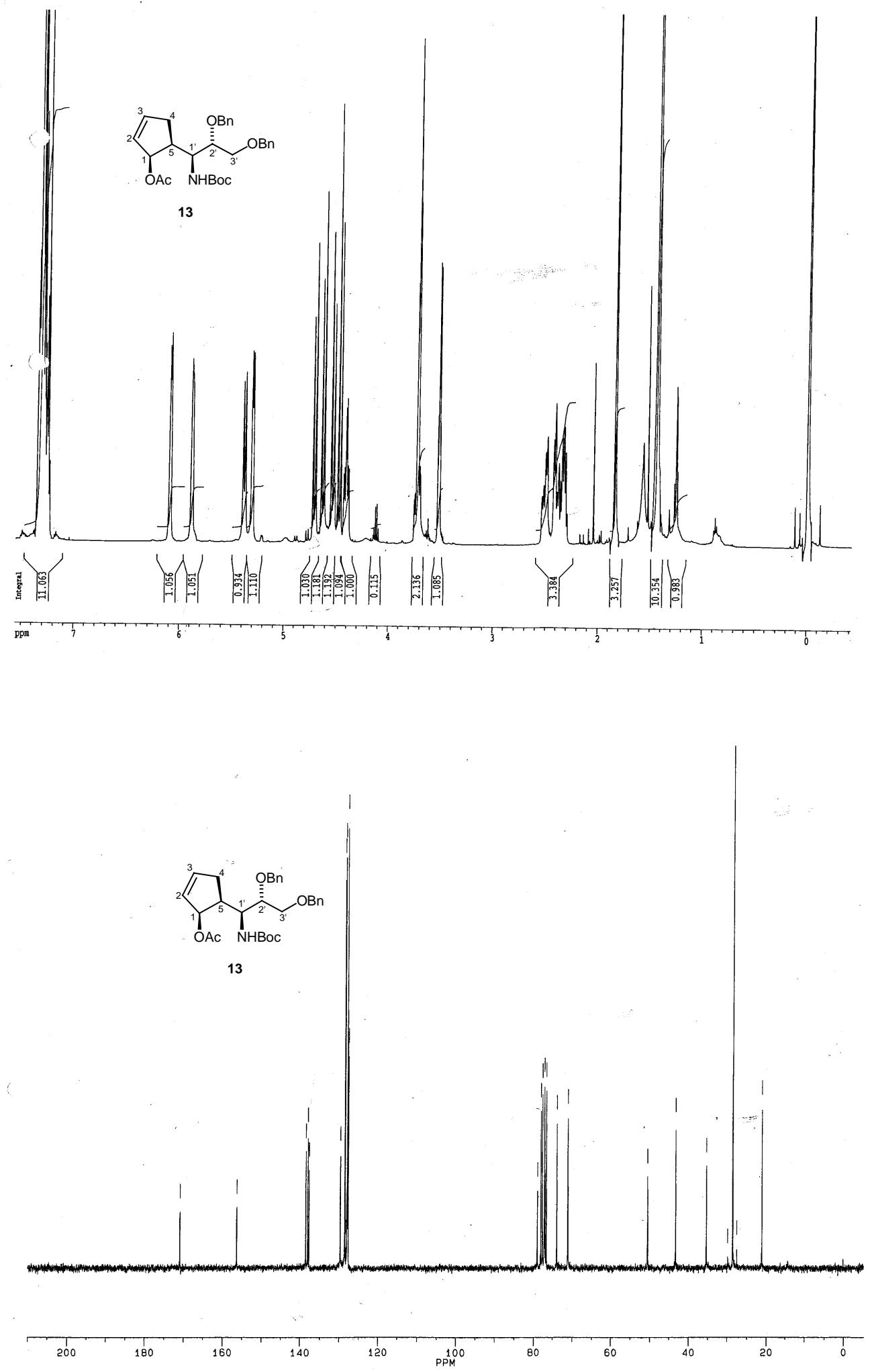
$\left(1 S, 2 S, 1^{\prime} R, 4^{\prime} R\right)-$

tert-butyl- [2,3-Bis-(benzyloxy)-1-(4'-methylcyclopent-2'-enyl)-propyl]carbamate (14):

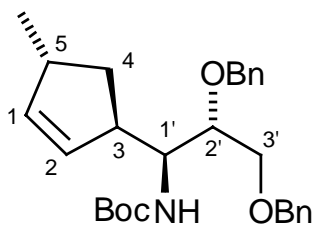

Copper(I)cyanide $(90.0 \mathrm{mg}, 1.01 \mathrm{mmol})$ was suspended in dry diethylether $(5 \mathrm{~mL})$ and cooled to - $20{ }^{\circ} \mathrm{C}$. To this was dropped a $3.0 \mathrm{~m}$ solution of methylmagnesium bromide in diethylether $(0.67$ $\mathrm{mL}, 2.02 \mathrm{mmol})$. After stirring for $20 \mathrm{~min}$, the solution of the acetate 13 (100 mg, $0.202 \mathrm{mmol})$ in dry diethylether $(5 \mathrm{~mL})$ was added dropwise over a periode of $5 \mathrm{~min}$ at $-20{ }^{\circ} \mathrm{C}$ and stirred for another $1.5 \mathrm{~h}$ at $-20{ }^{\circ} \mathrm{C}$. The reaction was quenched with saturated aqueous $\mathrm{NH}_{4} \mathrm{Cl}(5 \mathrm{~mL})$ and water ( $5 \mathrm{~mL})$. The organic phase was seperated and the aqueous layer was extracted with $\mathrm{CH}_{2} \mathrm{Cl}_{2}(4$ $\times 20 \mathrm{~mL})$. The combined organic phases were dried $\left(\mathrm{MgSO}_{4}\right)$ and the solvent was removed at reduced pressure. The residue (d.r. 88:12) was purified by flash chromatography on silica (petroleum ether/ethyl acetate 4:1) to afford the cyclopentenederivative $\mathbf{1 4}(80 \mathrm{mg}, 88 \%)$ as a colourless oil. 14: $[\alpha]_{D}^{20}=+65.7\left(c=0.77, \mathrm{CHCl}_{3}\right)$. IR (Film): $\widetilde{v}=1705,1494,1170,1085,1057$, 739, $693 \mathrm{~cm}^{-1} .{ }^{1} \mathrm{H}$ NMR $\left(300.1 \mathrm{MHz}, \mathrm{CDCl}_{3}\right): \delta=1.01\left(\mathrm{~d}, J_{5, \mathrm{CH} 3}=7.0 \mathrm{~Hz}, 3 \mathrm{H}, 5-\mathrm{CH}_{3}\right), 1.30(\mathrm{ddd}$, $\left.J_{3,4 \mathrm{a}}=5.3, J_{4 \mathrm{a}, 5}=8.4, J_{4 \mathrm{a}, 4 \mathrm{~b}}=13.1 \mathrm{~Hz}, 1 \mathrm{H}, 4-\mathrm{H}_{\mathrm{a}}\right), 1.45\left[\mathrm{~s}, 9 \mathrm{H}, \mathrm{C}\left(\mathrm{CH}_{3}\right)_{3}\right], 1.91\left(\mathrm{ddd}, J_{3,4 \mathrm{~b}}=8.2, J_{4 \mathrm{a}, 4 \mathrm{~b}}\right.$ $\left.=13.1, J_{4 \mathrm{~b}, 5}=4.8 \mathrm{~Hz}, 1 \mathrm{H}, 4-\mathrm{H}_{\mathrm{b}}\right), 2.75(\mathrm{~m}, 1 \mathrm{H}, 5-\mathrm{H}), 3.03(\mathrm{~m}, 1 \mathrm{H}, 3-\mathrm{H}), 3.56\left(\mathrm{ddd}, J_{1^{\prime}, 2^{\prime}}=7.0, J_{2^{\prime}, 3^{\prime} \mathrm{a}}\right.$ $\left.=5.1, J_{2^{\prime}, 3^{\prime} \mathrm{b}}=2.9 \mathrm{~Hz}, 1 \mathrm{H}, 2^{\prime}-\mathrm{H}\right), 3.63\left(\mathrm{dd}, J_{2^{\prime}, 3^{\prime} \mathrm{a}}=5.0, J_{3^{\prime} \mathrm{a}, 3^{\prime} \mathrm{b}}=10.6 \mathrm{~Hz}, 1 \mathrm{H}, 3^{\prime}-\mathrm{H}_{\mathrm{a}}\right), 3.73\left(\mathrm{dd}, J_{2^{\prime}, 3^{\prime} \mathrm{b}}=\right.$ $\left.2.9, J_{3^{\prime} \mathrm{a}, 3^{\prime} \mathrm{b}}=10.6 \mathrm{~Hz}, 1 \mathrm{H}, 3^{\prime}-\mathrm{H}_{\mathrm{b}}\right), 3.84\left(\mathrm{~d}, J_{1^{\prime}, 2^{\prime}}=6.6 \mathrm{~Hz}, 1 \mathrm{H}, 1^{\prime}-\mathrm{H}\right), 4.51\left(\mathrm{~s}, 2 \mathrm{H}, \mathrm{C}_{2} \mathrm{C}_{6} \mathrm{H}_{5}\right), 4.56(\mathrm{~A}$ von $\left.\mathrm{AB}, J_{\mathrm{A}, \mathrm{B}}=11.6 \mathrm{~Hz}, 1 \mathrm{H}, \underline{\mathrm{CH}}_{\mathrm{A}} \mathrm{CH}_{\mathrm{B}} \mathrm{C}_{6} \mathrm{H}_{5}\right), 4.66\left(\mathrm{~B}\right.$ von $\mathrm{AB}, J_{\mathrm{A}, \mathrm{B}}=11.6 \mathrm{~Hz}, 1 \mathrm{H}, \mathrm{CH}_{\mathrm{A}} \underline{\mathrm{H}}_{\mathrm{B}} \mathrm{C}_{6} \mathrm{H}_{5}$ ), 5.53 (d"t", $\left.J_{1,2}=5.7, J_{2,3}={ }^{4} J_{2,5}=2.3 \mathrm{~Hz}, 1 \mathrm{H}, 2-\mathrm{H}\right), 5.65$ (d"t", $J_{1,2}=5.7,{ }^{4} J_{1,3}=J_{1,5}=1.9 \mathrm{~Hz}, 1 \mathrm{H}$, 1-H), $6.29\left(\mathrm{bd}, J_{1, \mathrm{NH}}=10.1 \mathrm{~Hz}, \mathrm{NH}\right), 7.23-7.36\left(\mathrm{~m}\right.$, zus. $\left.10 \mathrm{H}, 2 \mathrm{C}_{6} \mathrm{H}_{5}\right) .{ }^{13} \mathrm{C}-\mathrm{NMR}(75.5 \mathrm{MHz}$, $\left.\mathrm{CDCl}_{3}\right): \delta=21.0\left(\mathrm{q}, 5-\mathrm{CH}_{3}\right), 28.4$ [q, $\left.\mathrm{C}\left(\mathrm{CH}_{3}\right)_{3}\right], 34.8$ (t, C-4), 38.9 (d, C-5), 47.1 (d, C-3), 54.1 (d, C-1'), 71.5, 71.8, 73.7 (3 t, C-3' und $\left.2 \underline{\mathrm{CH}}_{2} \mathrm{C}_{6} \mathrm{H}_{5}\right), 78.2$ (d, C-2'), 78.8 [s, $\left.\underline{\mathrm{C}}\left(\mathrm{CH}_{3}\right)_{3}\right], 127.5,127.7$, 127.8, 128.3, 128.4 (5 d, o-, $m-, p-\mathrm{C}$ von $\mathrm{C}_{6} \mathrm{H}_{5}$ ), 130.5 (d, C-2), 138.9 (d, C-1), 130.5, 138.9 (2 s, $i-$ $\mathrm{C}$ von $\mathrm{C}_{6} \mathrm{H}_{5}$ ), 156.3 (s, 1-NHCOO) ppm. $\mathrm{C}_{28} \mathrm{H}_{37} \mathrm{NO}_{4}$ (451.60): calcd. C 74.47, H 8.26, N 3.10; found C 74.77, H 8.55, N 2.98. 

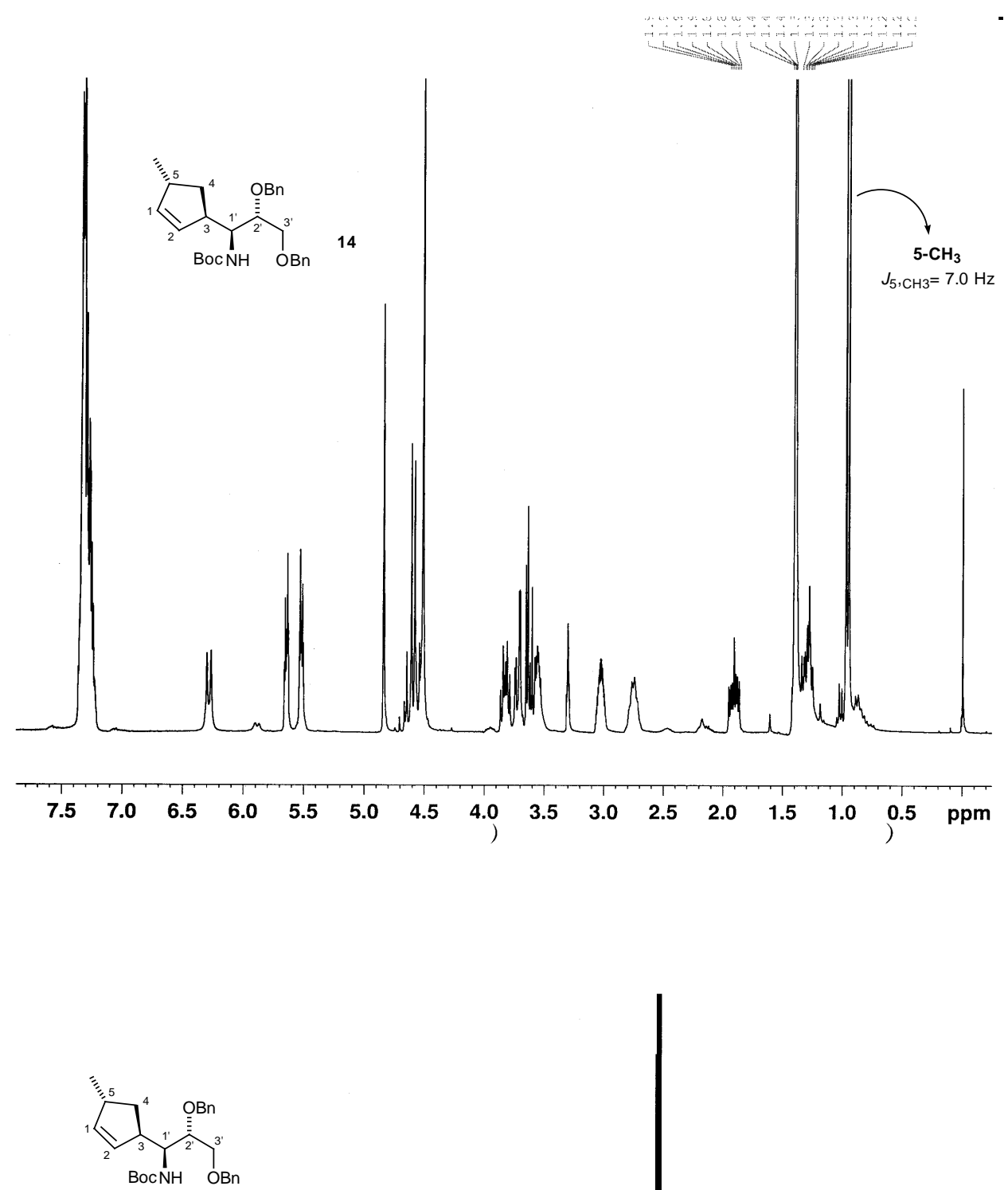

14

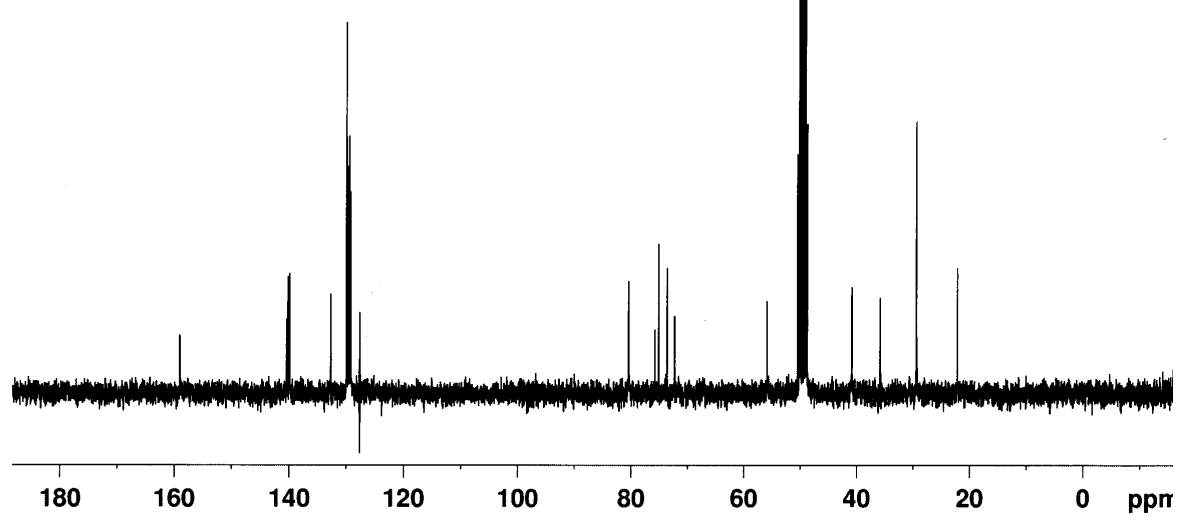




\section{(1S,2S,1'R,4'R)-tert-Butyl-[2,3-dihydroxy-1-(4'-methylcyclopent-2'-enyl)-propyl]-carbamate}

(15):

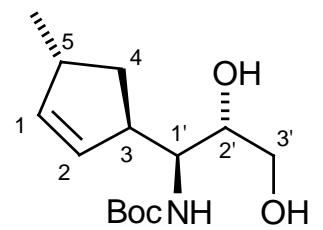

About $40 \mathrm{~mL}$ of liquid ammonia was placed in a round-bottomed flask put into a cooling bath at - $78{ }^{\circ} \mathrm{C}$. A solution of the benzyl ether $14(345 \mathrm{mg}, 0.764 \mathrm{mmol}$, d.r. 88:12) in THF (3 mL) was added under nitrogen in one portion, followed by sodium metal $(176 \mathrm{mg}, 7.64 \mathrm{mmol})$ in small pieces. After the appearance of a persistent blue colour the reaction mixture was stirred for $5 \mathrm{~s}$ and then quenched with methanol $(10 \mathrm{~mL})$. The mixture was warmed to room temp. and the residual ammonia evaporated by means of a stream of nitrogen. After neutralization with $1 \mathrm{~N} \mathrm{HCl}$, the mixture was extracted with $\mathrm{CH}_{2} \mathrm{Cl}_{2}(4 \times 20 \mathrm{~mL})$, and dried $\left(\mathrm{MgSO}_{4}\right)$. After concentration of the solvent under reduced pressure the residue was purified by flash chromatography on silica (petroleum ether/ethyl acetate 3:7) to provide the diol 15 (191 mg, $92 \%)$ as a colourless solid. The diastereomeric ratio was increased to $95: 5$ by two recrystallizations from $\mathrm{CH}_{2} \mathrm{Cl}_{2} /$ heptane. 15: M.p. $=68-72{ }^{\circ} \mathrm{C} \cdot[\alpha]_{D}^{20}=+154.8\left(c=0.775, \mathrm{CHCl}_{3}\right) . \mathrm{IR}: \tilde{v}=3340,2960,2930,1675,1515,1430,1355$, $1230,1158 \mathrm{~cm}^{-1} .{ }^{1} \mathrm{H}$ NMR $\left(300.1 \mathrm{MHz}, \mathrm{CD}_{3} \mathrm{OD}\right): \delta=1.00\left(\mathrm{~d}, J_{5, \mathrm{CH} 3}=6.9 \mathrm{~Hz}, 3 \mathrm{H}, 5-\mathrm{CH}_{3}\right), 1.41$ $\left[\mathrm{s}, 9 \mathrm{H}, \mathrm{C}\left(\mathrm{CH}_{3}\right)_{3}\right], 1.43\left(\mathrm{ddd}, J_{3,4 \mathrm{a}}=5.0, J_{4 \mathrm{a}, 5}=8.3, J_{4 \mathrm{a}, 4 \mathrm{~b}}=13.3 \mathrm{~Hz}, 1 \mathrm{H}, 4-\mathrm{H}_{\mathrm{a}}\right), 1.95\left(\mathrm{ddd}, J_{3,4 \mathrm{~b}}=\right.$ 8.7, $\left.J_{4 \mathrm{a}, 4 \mathrm{~b}}=13.6, J_{4 \mathrm{~b}, 5}=5.1, \mathrm{~Hz}, 1 \mathrm{H}, 4-\mathrm{H}_{\mathrm{b}}\right), 2.78(\mathrm{~m}, 1 \mathrm{H}, 5-\mathrm{H}), 3.16(\mathrm{~m}, 1 \mathrm{H}, 3-\mathrm{H}), 3.49,3.53$, 3.61, $3.66\left(4 \mathrm{~m}, 4 \mathrm{H}, 1^{\prime}-, 2^{\prime}-, 3^{\prime}-\mathrm{H}_{\mathrm{a}}\right.$ und 3'- $\left.\mathrm{H}_{\mathrm{b}}\right), 5.57$ (d"t", $\left.J_{1,2}=5.7, J_{2,3}={ }^{4} J_{2,5}=2.0 \mathrm{~Hz}, 1 \mathrm{H}, 2-\mathrm{H}\right)$, $5.66\left(\mathrm{~d} " \mathrm{t} ",{ }^{4} J_{1,3}=J_{1,5}=2.0, J_{1,2}=5.7 \mathrm{~Hz}, 1 \mathrm{H}, 1-\mathrm{H}\right) \mathrm{ppm} .{ }^{13} \mathrm{C} \mathrm{NMR}\left(75.5 \mathrm{MHz}, \mathrm{CDCl}_{3}\right): \delta=21.1$ (q, 5- $\left.\mathrm{CH}_{3}\right), 28.3$ [q, $\mathrm{C}\left(\underline{\mathrm{CH}}_{3}\right)_{3}$ ], 33.2 (t, C-4), 39.3 (C-5), 45.5 (d, C-3), 54.6 (d, C-1'), 63.1, (t, C-3'), 73.1 (d, C-2'), 80.4 [s, $\left.\underline{\mathrm{C}}\left(\mathrm{CH}_{3}\right)_{3}\right], 130.9$ (d, C-2), 138.7 (d, C-1), 157.8 (s, 1-NHCOO) ppm. $\mathrm{C}_{14} \mathrm{H}_{25} \mathrm{NO}_{4}$ (271.35): calcd. C 61.97, H 9.29, N 5.16; found C 62.06, H 9.27, N 5.00. 

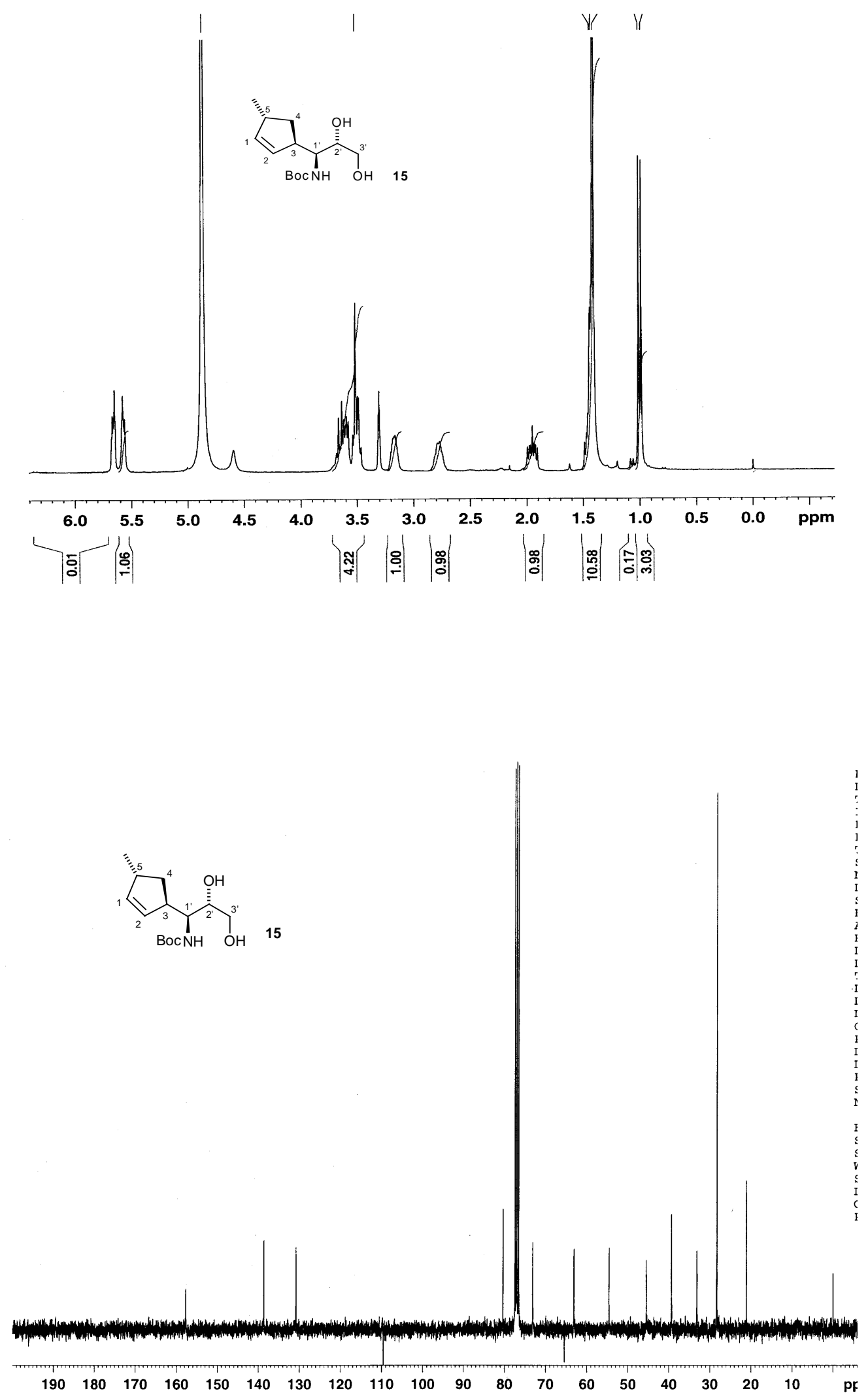


\section{Methyl-(2S,1'R,4'R)-tert-Butoxycarbonylamino-(4'-methylcyclopent-2'-enyl)-acetate, $N$-Boc-}

L-carbafuranomycin (16):

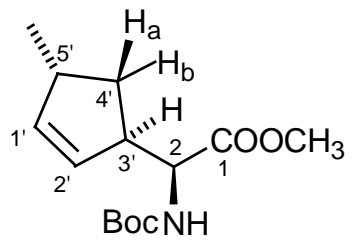

To a stirred solution of the diol $\mathbf{1 5}$ in $\mathrm{CH}_{2} \mathrm{Cl}_{2}(5 \mathrm{~mL})$ at $-20{ }^{\circ} \mathrm{C}$ was added lead tetraacetate (240 $\mathrm{mg}, 0.446 \mathrm{mmol}$, containing ca. $15 \%$ acetic acid as stabilizer). After stirring for $30 \mathrm{~min}$, TLC analysis indicated incomplete reaction, so some more lead tetraacetate $(22 \mathrm{mg}, 0.041 \mathrm{mmol})$ was added. The stirring was continued for $10 \mathrm{~min}$ and the mixture was filtered off. The removal of the solvent at $-20{ }^{\circ} \mathrm{C}$ in vacuo provided an oil, which was dissolved in tert-butylalcohol (3 mL), acetonitrile $(2 \mathrm{~mL})$ and 2-methyl-2-butene $(1 \mathrm{~mL})$ at $-20^{\circ} \mathrm{C}$. A solution of sodium chlorite $(69 \mathrm{mg}$, $0.61 \mathrm{mmol}$, ca. $80 \%$ purity) and sodium dihydrogenphosphate monohydrate ( $84 \mathrm{mg}, 0.61 \mathrm{mmol})$ in water $(1 \mathrm{~mL})$ was added dropwise over a period of $1 \mathrm{~h}$. After stirring for $5 \mathrm{~h}$ at $-20^{\circ} \mathrm{C}$, the mixture was filtered off and the solvent was removed under reduced pressure. After addition of water (15 $\mathrm{mL})$, dilute sulfuric acid was added $(\mathrm{pH} 2-3)$ and the mixture was extracted with diethylether $(5 \times$ $15 \mathrm{~mL})$. The organic solutions were washed with saturated aqueous sodium thiosulfate $(15 \mathrm{~mL})$ and dried $\left(\mathrm{MgSO}_{4}\right)$. After concentration of the solvent in vacuo, the residual oil was dissolved in diethyl ether $(10 \mathrm{~mL})$ at room temp., to which a solution of ca. $0.36 \mathrm{M}$ diazomethane $(5.6 \mathrm{~mL}$, ca. 2.0 mmol) in diethylether was added. After stirring for $10 \mathrm{~min}$ at room temp., the solvent was removed and the residue was purified by flash chromatography (petroleum ether/ethyl acetate 4:1) to afford ester the $16(84 \mathrm{mg}, 77 \%)$ as a colourless oil. 16: $[\alpha]_{D}^{20}=+156.4\left(c=1.01, \mathrm{CHCl}_{3}\right)$. IR (Film): $\tilde{v}=$ 2868, 1741, 1709, 1501, 1364, 1248, $1160 \mathrm{~cm}^{-1} .{ }^{1} \mathrm{H}$ NMR $\left(300.1 \mathrm{MHz}, \mathrm{CD}_{3} \mathrm{OD}\right): \delta=1.01(\mathrm{~d}$, $\left.J_{5^{\prime}, \mathrm{CH} 3}=7.0 \mathrm{~Hz}, 3 \mathrm{H}, 5^{\prime}-\mathrm{CH}_{3}\right), 1.43\left[\mathrm{~s}, 9 \mathrm{H}, \mathrm{C}\left(\mathrm{CH}_{3}\right)_{3}\right], 1.50\left(\mathrm{ddd}, J_{3^{\prime}, 4^{\prime} \mathrm{a}}=5.1, J_{4}{ }^{\prime} \mathrm{a}, 4^{\prime} \mathrm{b}=13.2, J_{4^{\prime} \mathrm{a}, 5^{\prime}}=\right.$ $\left.8.8 \mathrm{~Hz}, 1 \mathrm{H}, 4^{\prime}-\mathrm{H}_{\mathrm{a}}\right), 1.93\left(\mathrm{ddd}, J_{3^{\prime}, 4^{\prime} \mathrm{b}}=8.1, J_{4^{\prime} \mathrm{a}, 4^{\prime} \mathrm{b}}=13.2, J_{4^{\prime} \mathrm{b}, 5^{\prime}}=4.7 \mathrm{~Hz}, 1 \mathrm{H}, 4^{\prime}-\mathrm{H}_{\mathrm{b}}\right), 2.80(\mathrm{~m}, 1 \mathrm{H}$, 5'-H), 3.14 (m, 1 H, 3'-H), 3.71 (s, 3 H, $\mathrm{COOCH}_{3}$ ), 4.07 (d, J $\mathrm{J}_{2,3}$ ' = $\left.7.0 \mathrm{~Hz}, 1 \mathrm{H}, 2-\mathrm{H}\right), 5.53$ (d"t", $\left.J_{1^{\prime}, 2^{\prime}}=5.7, J_{2^{\prime}, 3^{\prime}}={ }^{4} J_{2^{\prime}, 5^{\prime}}=2.1 \mathrm{~Hz}, 1 \mathrm{H}, 2^{\prime}-\mathrm{H}\right), 5.74\left(\mathrm{~d}^{\prime \prime} \mathrm{t}^{\prime \prime}, J_{1^{\prime}, 2^{\prime}}=5.8,{ }^{4} J_{1^{\prime}, 3^{\prime}}=J_{1^{\prime}, 5^{\prime}}=2.1 \mathrm{~Hz}, 1 \mathrm{H}, 1^{\prime}-\right.$ H) ppm. ${ }^{13} \mathrm{C}$ NMR $\left(125.8 \mathrm{MHz}, \mathrm{CDCl}_{3}\right): \delta=21.1$ (q, 5'- $\left.\mathrm{CH}_{3}\right), 28.3$ [q, $\left.\mathrm{C}\left(\underline{\mathrm{CH}}_{3}\right)_{3}\right], 33.9$ (t, C-4'), 39.4 (C-5'), 48.5 (d, C-3'), 52.1 (q, $\left.\mathrm{OCH}_{3}\right), 56.4$ (d, C-2), 79.8 [s, $\left.\underline{\mathrm{C}}\left(\mathrm{CH}_{3}\right)_{3}\right], 129.1$ (d, C-2'), 139.6 (d, C-1'), 155.5 (s, 1-NHㅡO), 172.7 (s, $\underline{\mathrm{COOCH}}_{3}$ ) ppm. $\mathrm{C}_{14} \mathrm{H}_{23} \mathrm{NO}_{4}$ (269.34): calcd. C 62.43, H 8.61, N 5.20; found C 62.48, H 8.62, N 5.13. 


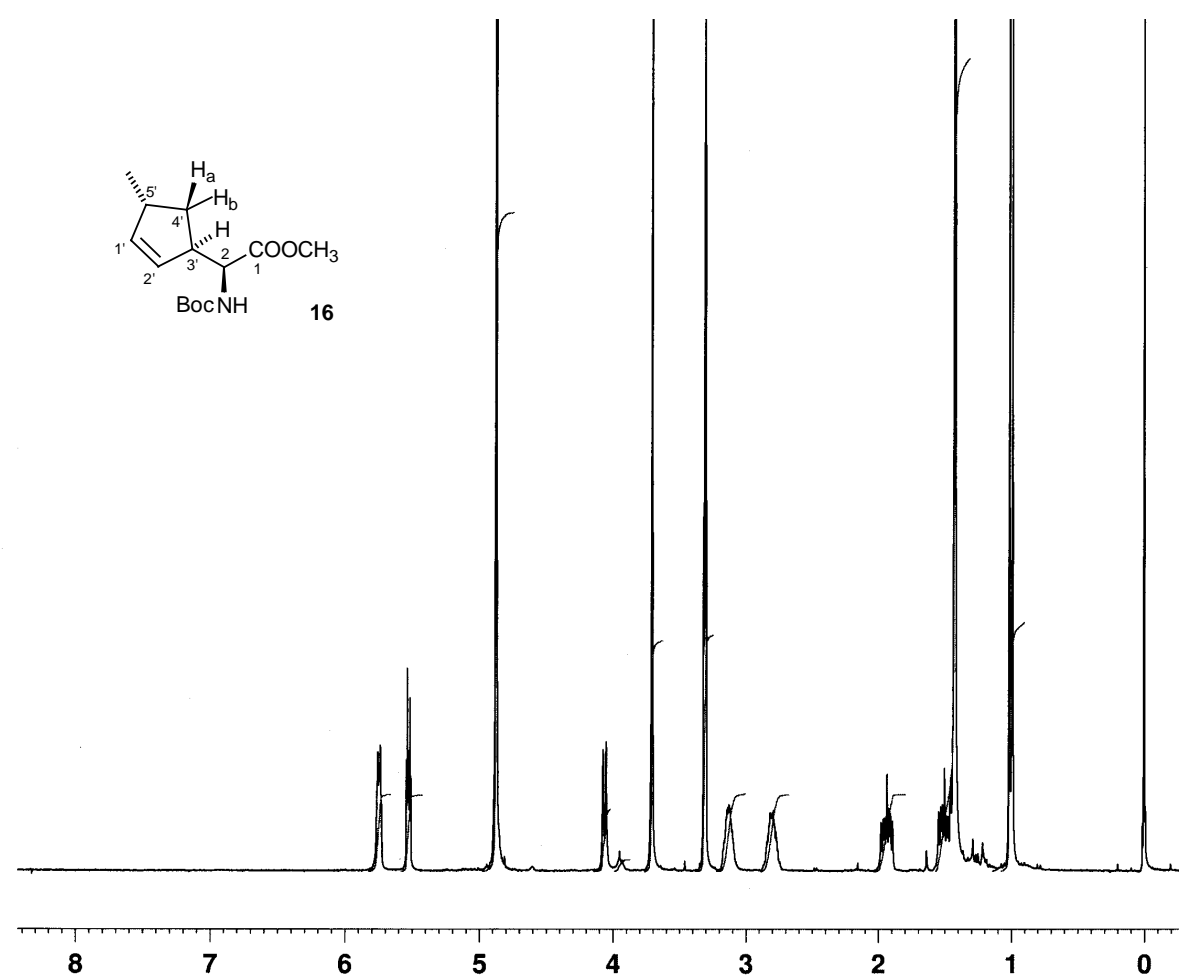




\section{(2S,1'R,4'R)-(4'-methylcyclopent-2'-enyl)-glycine, L-Carbafuranomycin (2):}

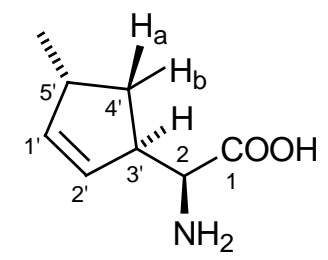

The ester 16 (17 mg, $0.063 \mathrm{mmol})$ was dissolved in $90 \%$ trifluoracetic acid (5 mL) at room temp. After stirring for $10 \mathrm{~min}$, TLC analysis indicated that the reaction had gone to completion. The solvent was removed under reduced pressure and co-evaporated with toluene $(2 \times 10 \mathrm{~mL})$. After removal of the solvent in vacuo $\left(10^{-2} \mathrm{mbar}, 0.5 \mathrm{~h}\right)$, the residue was dissolved in THF and $0.05 \mathrm{M}$ solution of $\mathrm{NaHCO}_{3}(2 \mathrm{~mL}) .0 .05 \mathrm{~m}$ solution of $\mathrm{NaOH}$ was added, until the $\mathrm{pH}$ of the solution was 10.5. After stirring for $2 \mathrm{~h}$ at room temp., conc. $\mathrm{HCl}(0.5 \mathrm{~mL})$ was added and the solvent was removed under reduced pressure. The residue was put on column with ion-exchange resin and eluted with $1_{\mathrm{N}}$ aqueous ammonia $(50 \mathrm{~mL})$ to afford a colourless solid, which was crystallized from acetone/water to provide the $\alpha$-amino acid $2(6.0 \mathrm{mg}, 61 \%)$ as a colourless solid. 2: M.p. 215$217^{\circ} \mathrm{C} \cdot[\alpha]_{D}^{20}=+164\left(c=0.08, \mathrm{H}_{2} \mathrm{O}\right)$. IR (neat): $\tilde{v}=3200-2500(\mathrm{~b}), 2950,2615,1605,1580$, 1509, 1448, 1418, 1392, $1156 \mathrm{~cm}^{-1}$. ${ }^{1} \mathrm{HNMR}$ (250.1 MHz, $\mathrm{D}_{2} \mathrm{O}$, intern standard: sodium-3trimethylsilylpropionate) $: \delta=1.03\left(\mathrm{~d}, J_{5^{\prime}, \mathrm{Me}}=7.0 \mathrm{~Hz}, 3 \mathrm{H}, 5^{\prime}-\mathrm{CH}_{3}\right), 1.60\left(\mathrm{ddd}, J_{3^{\prime}, 4^{\prime} \mathrm{a}}=5.1, J_{4^{\prime} \mathrm{a}, 4^{\prime} \mathrm{b}}=\right.$ $\left.13.2, J_{4^{\prime}, 5^{\prime}}=8.8 \mathrm{~Hz}, 1 \mathrm{H}, 4^{\prime}-\mathrm{H}_{\mathrm{a}}\right), 1.89\left(\mathrm{ddd}, J_{3^{\prime}, 4^{\prime} \mathrm{b}}=8.1, J_{4^{\prime} \mathrm{a}, 4^{\prime} \mathrm{b}}=13.2, J_{4^{\prime} \mathrm{b}, 5^{\prime}}=4.7 \mathrm{~Hz}, 1 \mathrm{H}, 4^{\prime}-\mathrm{H}_{\mathrm{b}}\right)$, 2.82 (m, 1 H, 5'-H), 3.47 (m, 1 H, 3'-H), 3.80 (d, J J,3' = 4.5 Hz, 1 H, 2-H), 5.61 (d"t", $J_{1^{\prime}, 2^{\prime}}=5.7$, $\left.J_{2^{\prime}, 3^{\prime}}={ }^{4} J_{2^{\prime}, 5^{\prime}}=2.2 \mathrm{~Hz}, 1 \mathrm{H}, 2^{\prime}-\mathrm{H}\right), 5.97\left(\mathrm{~d}^{\prime \prime} \mathrm{t}^{\prime}, J_{1^{\prime}, 2^{\prime}}=5.7,{ }^{4} J_{1^{\prime}, 3^{\prime}}=J_{1^{\prime}, 5^{\prime}}=2.1 \mathrm{~Hz}, 1 \mathrm{H}, 1^{\prime}-\mathrm{H}\right) \mathrm{ppm}$. ${ }^{13} \mathrm{C}-\mathrm{NMR}\left(62.9 \mathrm{MHz}, \mathrm{D}_{2} \mathrm{O}\right): \delta=23.2$ (q, 5'- $\mathrm{CH}_{3}$ ), 34.9 (t, C-4'), 42.1 (d, C-5'), 49.0 (d, C-3'), 60.3 (d, C-2), 130.5 (d, C-2'), 144.8 (d, C-1'), 176.9 (s, COOH) ppm. MS (CI pos., $\left.\mathrm{CH}_{4}, 455 \mathrm{~K}\right): \mathrm{m} / \mathrm{z}=$ $156[\mathrm{M}+\mathrm{H}]^{+}$. HRMS (CI pos.): $[\mathrm{M}+\mathrm{H}]^{+} \mathrm{C}_{8} \mathrm{H}_{13} \mathrm{NO}_{2}$ calcd. 156.1025, found 156.1025. 

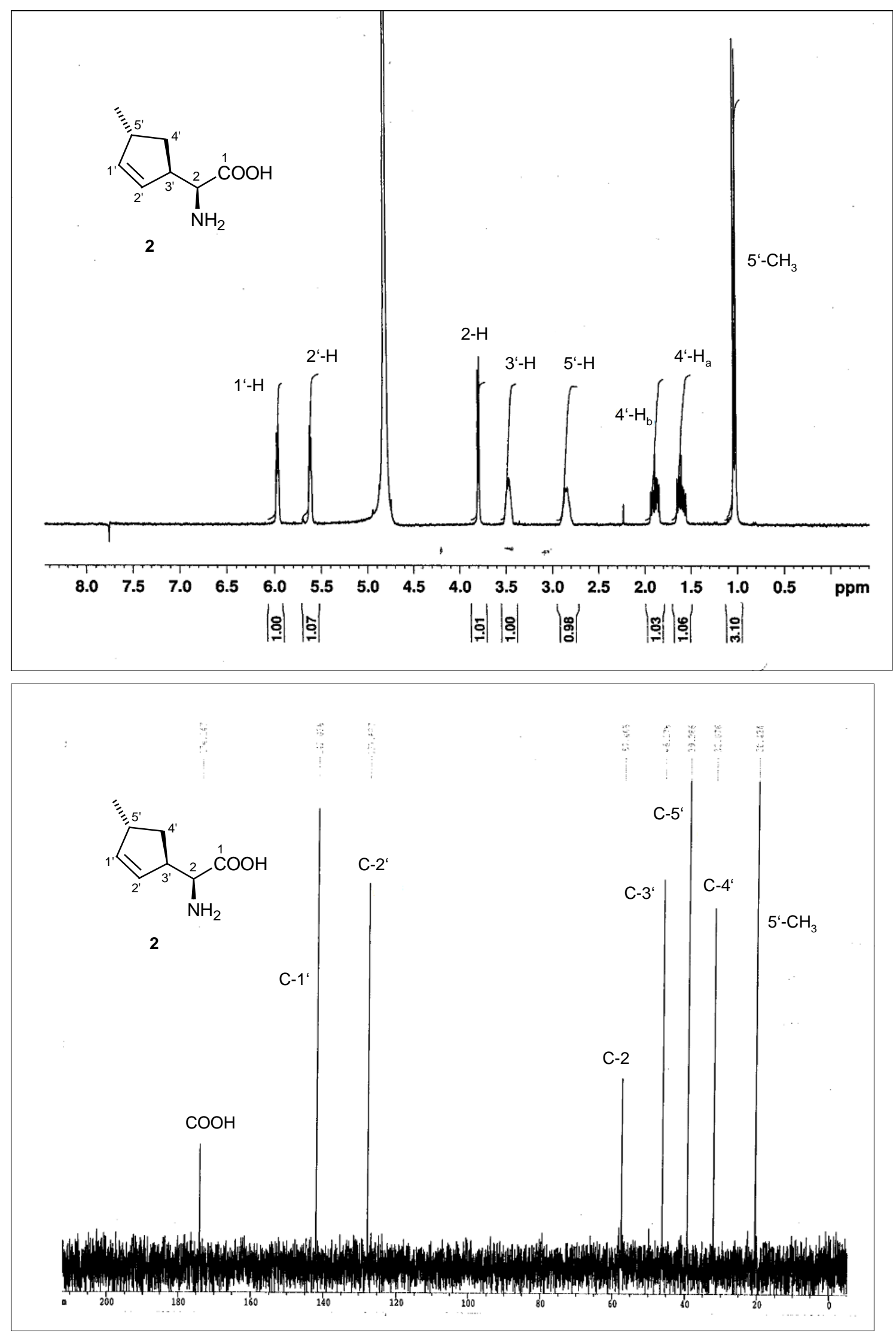